\title{
Téoros
}

Revue de recherche en tourisme

\section{Une carte du patrimoine religieux du Québec} 100 destinations

\section{Jean Simard}

Volume 16, numéro 2, été 1997

Tourisme et religion

URI : https://id.erudit.org/iderudit/1074578ar

DOI : https://doi.org/10.7202/1074578ar

Aller au sommaire du numéro

Éditeur(s)

Université du Québec à Montréal

ISSN

0712-8657 (imprimé)

1923-2705 (numérique)

Découvrir la revue

Citer cet article

Simard, J. (1997). Une carte du patrimoine religieux du Québec : 100

destinations. Téoros, 16(2), 34-37. https://doi.org/10.7202/1074578ar d'utilisation que vous pouvez consulter en ligne.

https://apropos.erudit.org/fr/usagers/politique-dutilisation/ 


\title{
UNE CARTE DU PATRIMOINE RELIGIEUX DU QUÉBEC
}

\section{DESTINATIONS}

\author{
Jean Simard, professeur \\ Département d'histoire - Université Laval, Québec
}

La carte du patrimoine religieux du Québec mériterait qu'on la dessine car son contenu ferait comprendre jusqu'à quel point la vallée du Saint-Laurent et ses prolongements sont investis par le sacré : près de 3000 églises catholiques et protestantes, presque autant de presbytères et de cimetières. À l'époque où l'Église catholique s'occupait d'éducation et de sante publiques, on a pu dénombrer plus de 1100 couvents, monastères, hôpitaux, collèges, sans compter les écoles. Ces structures existent toujours, même converties. La culture populaire a aussi laissé ses traces si l'on considère la situation des routes rurales qui comptent pas moins de 3500 croix et calvaires, en plus d'un millier de chapelles et d'oratoines qui ont résisté aux décrets du dernier concile. Les représentants de la nation ne sont pas restés insensibles à ce phénomène de culture. La Commission des monuments historiques (aujourd"hui Commission des biens culturels), fondée en 1922, entreprit d'en dresser les premiers inventaires l'année de sa création. Depuis ce temps, elle a classé ou reconnu 407 biens du patrimoine religieux, ce qui représente près de $50 \%$ de l'ensemble des biens culturels protégés par la loi québécoise. Il s'agit de 221 bâtiments (églises, chapelles, sanctuaires, presbytères), de 51 calvaires et croix de chemin, de 22 cimetières et charniers, de 53 collections d'auvres d'art et de 60 fonds d'archives de l'état civil (actes de baptêmes, de mariages et de sépultures)'. La carte du patrimoine religieux, disions-nous, reste à faire. Contentons-nous pour le moment de suggérer des chemins et des destinations que peuvent déjà suivre les citoyens et les visiteurs désireux de remonter aux sources de l'identité.

La montagne est certainement un lieu de prédilection pour la prière et plusieurs ont servi à cette fin. À Oka, au nord-ouest de Montréal, au sommet d'une montagne peuplée d'érables et de chênes rouges qui domine la région du lac des DeuxMontagnes, se dressent sept édicules en maçonnerie et en bardeaux qui forment entre eux un chemin de croix connu sous le nom de Calvaire d'Oka. Érigés en 1740 par les sulpiciens à l'intention des Amérindiens qui y montaient en pellerinage, ces édicules étaient garnis de sept peintures importées de France. Menacées par l'humidité et le froid, les peintures furent retirées des oratoires vers $1776 \mathrm{et}$ transférées dans l'église paroissiale
d'Oka où elles sont toujours. On les remplaça par des hauts-reliefs en bois polychrome commandés à François Guernon dit Belleville et à Louis-Amable Quévillon. Les reliefs restèrent là pendant deux siècles quand, en 1974, des inconnus mutilèrent la $5^{t}$ et la $7^{*}$ stations. Ils prirent à leur tour le chemin de l'église paroissiale où l'on peut maintenant les admirer.

Près de Chicoutimi, au sanctuaire de LacBouchette, on peut voir un chemin de croix traditionnel de 14 stations, sculpté au stylet dans la pierre de chaux de SaintMarc-des-Carrières. Il est l'auvre de R. Goffin de Chicoutimi, associé de la maison Delwaide et Goffin « manufac- turiers de monuments, exhibits et bureaux * de la rue Saint-Vallier à Québec. Il est placé non loin de la première chapelle du sanctuaire qu'a peinte Charles Huot de 1908 à 1920 , tout près aussi du calvaire de Louis Jobin (6 personnages) livré en 1918. Le chemin de croix fut inauguré et bénit le 15 août 1921 en présence de quelque 2000 personnes par le fondateur du sanctuaire, l'abbé Elzéar Delamarre, oncle de Victor Delamarre. Ce dernier était reconnu dans tout le Québec pour sa grande force physique autant que pour sa foi inébranlable qu'il mettait au service de son oncle. Ainsi, par exemple, lorsque l'abbé décida d'installer une statue de SaintMichel archange sur un rocher pyramidal d'environ cinq mètres de haut, alors qu'il n'avait ni grue ni palan, il demanda à Victor de l'aider. Dans un écrit qu'il consacre aux exploits de son neveu, l'abbé Delamarre raconte : $\approx$ On amena dans sa boite d'emballage la statue jusqu' au sommet de la pyramide où il fallait la placer. On la dressa debout, mais il lui restait à franchir, entre la plate-forme où elle était et le piédestal, un espace d'une couple de pieds dans le vide. Victor se plaça dans

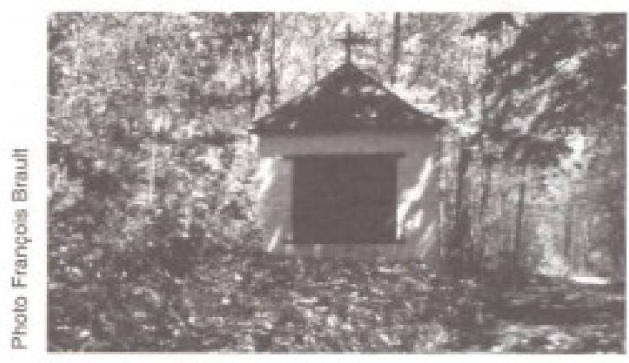

Calvaire d'Oka, 1740. L'un des sept édicules qui abritaient jusqu'en 1974 un chemin de croix. Le sentier qui conduit au calvaire et l'en= towre totalise $5,5 \mathrm{~km}$. Classe site historique en 1982. 


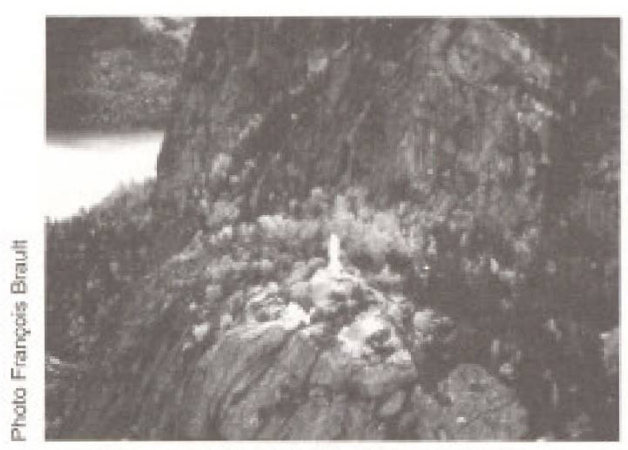

Notre-Dame-du-Saguenay sculpté par Louis Jobin en 1881. Sütuce sur la première corniche du cap Eternité, à $I I \mathrm{~km}$ de Rivière-Éternité, La statue est accessible au public qui doit emprunter un sentier escarpé de 3,5 km. Classée auvre d'art en 1965.

l'espace vide, $s$ 'y affermit les pieds sur le rebord du rocher, prit dans ses bras la statue par le socle et, prestement, la mit en place. D'après les sculpteurs qui l'ont taillée, le poids de la statue est d'au moins 1200 livres $*$ (Dragon, 1974 : 148-149).

Près de là, le paysage du cap Trinité aux falaises abruptes qui se jettent dans le Saguenay est plus grandiose encore. C'est au sommet de ce cap que, le 15 septembre 1881 , on inaugura la statue dite de Notre-Dame-du-Saguenay qui mesure 7,5 mètres de haut et pèse plus de 3000 kilos. Sculptée dans trois blocs de pin et recouverte ensuite de feuilles de plomb, la statue résiste encore au temps. Elle est l'ouvre de Louis Jobin. Six ans avant que Bartholdi n'installe sa Lady Liberty dans. le port de New York, Jobin aura sculpté, transporté, érigé et plombé, à une altitude de 180 mètres, la plus gigantesque statue exécutée en Amérique du Nord. Tombé malade alors qu'il remontait le Saguenay vers 1870 pour exercer ses activités de commis-voyageur, Charles-Napoléon Robitaille avait fait le vou d'offrir une statue à la Vierge si jamais elle lui accordait dix autres années de vie. Guéri, le commis-voyageur lança une campagne publique dans les journaux afin de financer cette entreprise * autant patriotique que religieuse $\%$.

Jacques Panet, qui fut de 1779 à 1829 curé de la paroisse Notre-Dame-deBonsecours de l'Islet-sur-Mer, à l'est de Québec, fit un geste tout aussi religieux et patriotique lorsqu'il commanda à l'orfèvre François Ranvoyzé ce que nous nommons aujourd'hui * le trésor de
l'Islet *. Ayant accumulé assez de louis d'or américains pour les faire couler en forme de calice et patène, de ciboire et d'ostensoir, le curé Panet écrivait dans son journal intime :

Il est honteux de voir que ni dans les toute l'Europe, et j'ose même assurer dans tout l'univers, on ne pourra trouver ces trois vases d'or, ni même un teux de voir que des prêtres riches et catholiques achètent des montres en or, des tabatières d'or lorsque l'on ne voit aucun vase d'or dans les églises (Simard, $1989: 179$ ).

Lorsque, en 1810 et en 1812, François Ranvoyzé livre sa commande au curé Panet, il trouve à l'Islet une église dont le chour vient d'être refait par François Baillairgé et son père Jean, à la mode du style Louis XVI. Le retable met en évidence le plus beau tableau du curé JeanAntoine Aide-Créquy, une huile sur toile de 1776 représentant L'Annonciation, tandis qu'un tabernacle de Noël Levasseur, fabriqué vers 1730 , en constitue la base. C'est dans ce tabernacle, entièrement doré à la feuille, que prennent place les ouvres de l'orfèvre.

Ailleurs, l'influence du curé se fera plutôt sentir sur le plan de l'architecture. L'abbe Pierre Conefroy, curé de la paroisse de la Sainte-Famille, à Boucherville sur la rive sud de Montréal, imagine deux Canadas, ni à Rome, ni dans seul calice d'or. Il est encore plus hon-

un nouveau plan d'église qui met ensemble la tradition issue de la NouvelleFrance et l'apport néo-classique des Britanniques. Comme on peut le voir à Boucherville, mais aussi à L'Acadie, dans des églises construites en 1801 selon le devis de Conefroy, l'influence néo-classique est manifeste dans les portails en pierre de taille et dans le fronton triangulaire qui en ornent les façades. A l'intérieur de l'église de Boucherville se marient par ailleurs les traditions esthétiques opposées de Baillairgé (Québec) et de Quévillon (Montréal).

Les $\mathrm{XVIII}^{*}$ et $\mathrm{XIX}^{*}$ siècles ne détiennent pas le monopole de la réussite en matière d'art religieux. Pour s'en convaincre, l'amateur pourra se rendre dans les lieux. décorés par Ozias Leduc dans la première

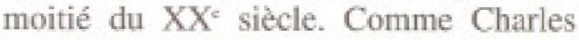
Huot l'avait fait un peu plus tôt au sanctuaire de Lac-Bouchette, Leduc entreprend en 1922 une commande dans laquelle il pourra faire valoir son talent. L'archevêque de Sherbrooke, M ${ }^{r}$ Paul LaRocque, voulait décorer la chapelle de son palais épiscopal autour d'un thème unique qui était celui de Marie corédemptrice. Quatre grands tableaux expliquent symboliquement le rôle de Marie dans l'auvre rédemptrice de son fils. Tout d'abord L'Annonce de Marie coredemptrice et L'Annonciation, qui mettent en évidence le rôle historique de la Vierge dans le salut des hommes, puis $L a$ Présentation de Jésus au temple et La Crucifixion où Jésus assume seul le

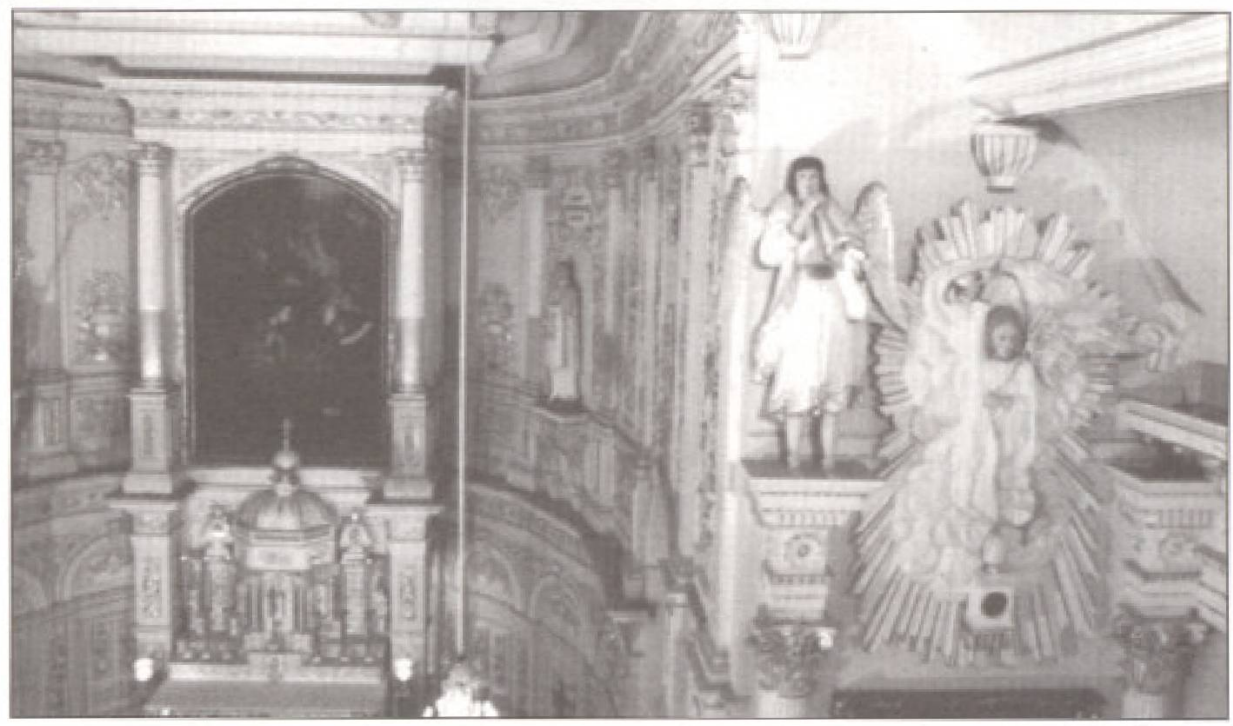

Chaur de l'église de l'Islet-sur-Mer. Le retable principal fut entrepris par François Baillairgé et son père Jean en 1782, Les bas-reliefs couronnant les retables latéraux sont de Pierre-Florent Baillairgé, frère de François, qui les sculpta en 1807. L'ange à la trompette fut exéculé par Amable Charron en 1815. Eglise classée monument historique en 1957. 
fardeau de la preuve. La réussite de cet ouvrage s'explique en grande partie par l'entente harmonieuse entre l'archevếque et le peintre.

Notre-Dame-de-la-Présentation de Shawinigan-Sud sera la dernière aeuvre de Leduc. C'est en 1943 et il a 79 ans. Euvre originale, aeuvre de méditation et d'harmonie aussi entre la recherche de l'universel, l'enracinement dans l'histoire et l'ancrage dans le milieu des travailleurs de $1^{+}$agriculture et de l'industrie, la décoration de cette église a encore pour theme la Rédemption. On ne pouvait choisir meilleure thematique pour interpreter le genre de vie de cette population occupée à la transformation des ressources naturelles après dix années de crise economique. Il y consacre quatre tableaux : l'agriculture, la foresterie, la transformation du bois en papier et le coulage de l'aluminium. Le peintre s'éteint le 16 juin 1955 avant d'avoir terminé son contrat. C'est son êlève, Gabrielle Messier, quil achève seule le dernier tableau esquissé par le maître. Les visiteurs du pare de la Cité de l'énergie à Shawinigan auraient raison de faire un détour par Shawinigan-Sud avant de rentrer.

Qu'il parte de Montréal, de Sherbrooke, de Hull, de Rouyn-Noranda, de TroisRivières, de Québec, de Chicoutimi ou de Rimouski, le voyageur ne manque pas de destinations pour satisfaire son gout de l'architecture et de l'art religieux. J'en propose 100 dans la liste qui suit. Il s'agit d'un choix personnel qui n'a rien de proprement scientifique. Je tente néanmoins d'y représenter l'ensemble des régions, les villes et les campagnes, l'art populaire comme le plus savant. Surtout je classe les lieux à visiter en trois catégories. Ceux qui ont une croisette (50) ont un intếrêt patrimonial indiscutable ; ceux qui en ont deux (41) peuvent être considérés comme appartenant à l'essentiel du patrimoine religieux ; ceux enfin qui en ont trois (9) forment évidemment la liste des incontournables. Les critères d'évaluation utilisés pour départager les destinations en nombre de croisettes sont ceux qu'utilisent en général les spécialistes : caractéristiques architecturales, patrimoine artistique, qualités paysagères, intêrêt historique ou symbolique. Mais ces critères sont pondérés ici à ma manière.

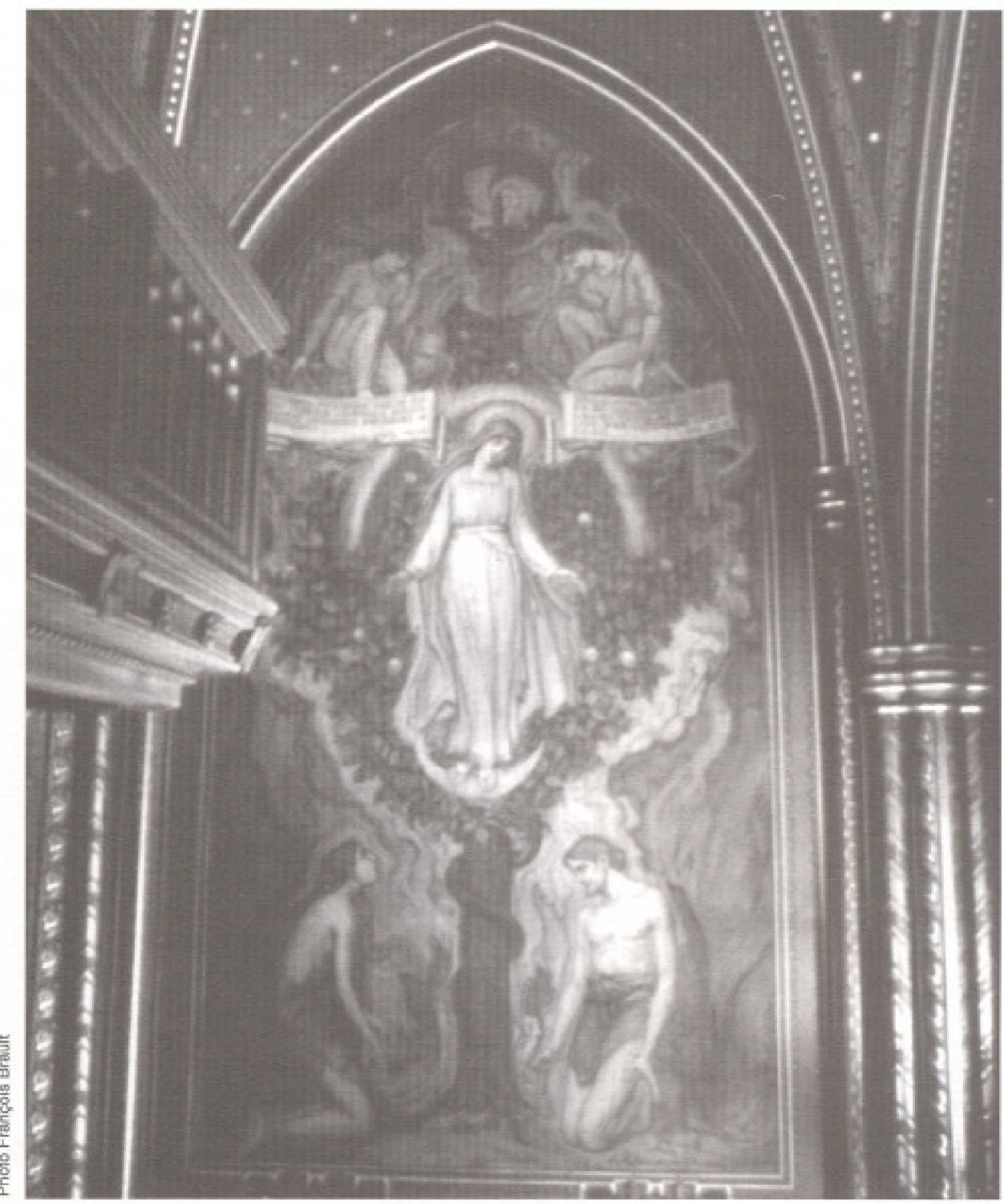

Chapelle privée de l'archevêché de Sherbrooke. Tableau de L'Annonce de Marie cordenptrice peint par Ozias Leduc entre 1922 et 1932. Le décor intérieur concu et ríalisé par Leduc transformera cette toute petite chapelle en une sorte d'écrin. Classée auvre d'art en 1993.

\section{NOTE}

I Ces chiffres sont tirés de : Lauzon, 1994; Commission des biens culturels, 1995 ; et Simard, 1995.

\section{BIBLIOGRAPHIE}

Commission des biens culturels du Québec (1995), * Le Patrimoine religicux : le temps d'agir $\%$, Patrimoine, Québec, 1, 3, automne

Dragon, A. (1974), L'abbé Delamarre, fondateur des sours antoniennes de Marie et des pelerinages du lac Bouchette. Chicoutimi, Société historique du Saguenay, p. 148-149.

Lauzon, Danicl (1994), Bilan des interventions du ministère de la Culture et des Communi- cations du Qubbec pour diverses categories de biens culturels, Québec, Ministère de la Culture et des Communications, dossiers 6241, 6901$6903,6910-1915,6920,6922,6930)$.

Simard, Jean (1995), Liart religieux des routes du Quebec, Québec, les Publications du Québec (Lieux et traditions - 6).

Simard, Jean (1989), Les Arts sacrés au Ouebec, Boucherville, Editions de Mortagne, p. 179. 


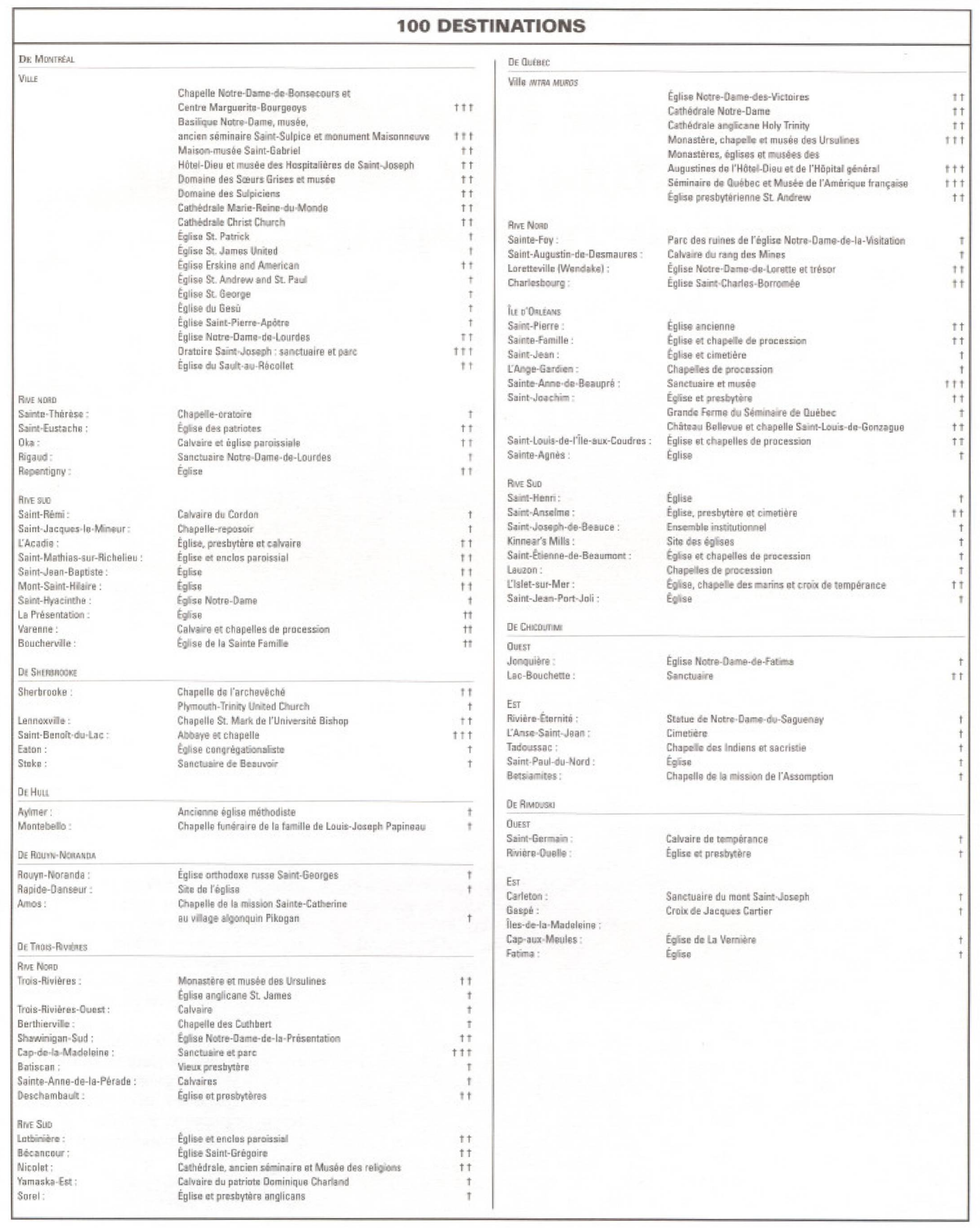

